\title{
Classification of Traumatic Brain Injury for Targeted Therapies
}

\author{
KATHRYN E. SAATMAN, ${ }^{1}$ ANN-CHRISTINE DUHAIME, ${ }^{2}$ ROSS BULLOCK, ${ }^{3}$ \\ ANDREW I.R. MAAS, ${ }^{4}$ ALEX VALADKA, ${ }^{5}$ and GEOFFREY T. MANLEY, ${ }^{*} *$ \\ and WORKSHOP SCIENTIFIC TEAM AND ADVISORY PANEL MEMBERS*
}

\begin{abstract}
The heterogeneity of traumatic brain injury (TBI) is considered one of the most significant barriers to finding effective therapeutic interventions. In October, 2007, the National Institute of Neurological Disorders and Stroke, with support from the Brain Injury Association of America, the Defense and Veterans Brain Injury Center, and the National Institute of Disability and Rehabilitation Research, convened a workshop to outline the steps needed to develop a reliable, efficient and valid classification system for TBI that could be used to link specific patterns of brain and neurovascular injury with appropriate therapeutic interventions. Currently, the Glasgow Coma Scale (GCS) is the primary selection criterion for inclusion in most TBI clinical trials. While the GCS is extremely useful in the clinical management and prognosis of TBI, it does not provide specific information about the pathophysiologic mechanisms which are responsible for neurological deficits and targeted by interventions. On the premise that brain injuries with similar pathoanatomic features are likely to share common pathophysiologic mechanisms, participants proposed that a new, multidimensional classification system should be developed for TBI clinical trials. It was agreed that preclinical mod-
\end{abstract}

\footnotetext{
*Workshop Scientific Team and Advisory Panel, including: David Brody, ${ }^{7}$ Charles Contant, ${ }^{8}$ Pramod Dash, ${ }^{9}$ Ramon DiazArrastia, ${ }^{10}$ Stephanie Fertig, ${ }^{11}$ Alisa Gean, ${ }^{6}$ Clay Goodman, ${ }^{5}$ Wayne Gordon, ${ }^{12}$ Ronald Hayes, ${ }^{13}$ Ramona Hicks, ${ }^{11}$ Jean Langloi, ${ }^{14}$ Anthony Marmarou, ${ }^{15}$ David Moore,${ }^{16}$ Gordon Murray, ${ }^{17}$ David Okonkwo, ${ }^{18}$ Linda Papa,${ }^{19}$ Linda Phillips, ${ }^{15}$ Nikolaus Plesnila, ${ }^{20}$ Claudia Robertson, ${ }^{5}$ Courtney Robertson, ${ }^{21}$ Juan Sahuquillo, ${ }^{22}$ Robert Silbergleit, ${ }^{23}$ Ewout Steyerberg, ${ }^{24}$ Nino Stocchetti, ${ }^{25}$ Evelyn Teasdale, ${ }^{26}$ Graham Teasdale, ${ }^{27}$ Nancy Temkin, ${ }^{28}$ Hilaire Thompson, ${ }^{28}$ Karen Tong, ${ }^{29}$ Lindsay Wilson, ${ }^{30}$ David Wright. ${ }^{31}$

${ }^{1}$ University of Kentucky, Lexington, KY, ${ }^{2}$ Dartmouth Hitchcock Medical Center, Lebanon, NH, ${ }^{3}$ University of Miami, Miami, FL, ${ }^{4}$ University Hospital Antwerp, Belgium, ${ }^{5}$ University of Texas Medical School at Houston, Houston, TX, ${ }^{6}$ University of California, San Francisco, CA, ${ }^{7}$ Washington University, School of Medicine, St. Louis, MO, ${ }^{8}$ Pfizer Pharmaceutical Company, Groton, CT, ${ }^{9}$ University of Texas Medical Center, Houston, TX, ${ }^{10}$ University of Texas Southwestern, Medical Center, Dallas, TX, ${ }^{11}$ National Institute of Neurological Disorders and Stroke, Bethesda, MD, ${ }^{12}$ Mount Sinai School of Medicine, New York, NY, ${ }^{13}$ Banyan Biomarkers, Inc., Gainesville, FL, ${ }^{14}$ National Center for Injury Prevention and Control, Atlanta, GA, ${ }^{15}$ Virginia Commonwealth University, Medical College of Virginia, Richmond, VA, ${ }^{16}$ Defense and Veterans Brain Injury Center (DVBICHQ), Washington, DC, ${ }^{17}$ University of Edinburgh, Edinburgh, United Kingdom, ${ }^{18}$ University of Pittsburgh Medical Center, Pittsburgh, PA, ${ }^{19}$ University of Florida, Gainesville, FL, ${ }^{20}$ University of Munich Medical Center, Munich, Germany, ${ }^{21}$ University of Maryland, Baltimore, MD, ${ }^{22}$ Vall d'Hebron University Hospital, Barcelona, Spain, ${ }^{23}$ University of Michigan, Ann Arbor, MI ${ }^{24}$ Erasmus MC-University Medical Center, Rotterdam, Netherlands, ${ }^{25}$ Universita di Milano, Terapia Intensiva Neuroscienze, Milano, Italy, ${ }^{26}$ Institute of Neurological Sciences, South Glasgow University NHS Hospital, and ${ }^{27}$ NHS Quality Improvement Scotland, Glasgow, Scotland, ${ }^{28}$ University of Washington, Seattle, WA, ${ }^{29}$ Loma Linda University, Loma Linda, CA ${ }^{30}$ University of Stirling, Stirling, United Kingdom, ${ }^{31}$ Emory University, Atlanta, GA.
} 


\section{SAATMAN ET AL.}

els were vital in establishing pathophysiologic mechanisms relevant to specific pathoanatomic types of TBI and verifying that a given therapeutic approach improves outcome in these targeted TBI types. In a clinical trial, patients with the targeted pathoanatomic injury type would be selected using an initial diagnostic entry criterion, including their severity of injury. Coexisting brain injury types would be identified and multivariate prognostic modeling used for refinement of inclusion/exclusion criteria and patient stratification. Outcome assessment would utilize endpoints relevant to the targeted injury type. Advantages and disadvantages of currently available diagnostic, monitoring, and assessment tools were discussed. Recommendations were made for enhancing the utility of available or emerging tools in order to facilitate implementation of a pathoanatomic classification approach for clinical trials.

Key words: clinical trial; head injury; intervention; outcome; therapy

\section{INTRODUCTION}

$\mathbf{T}$ RAUMATIC BRAIN INJURY (TBI) remains a major cause of death and disability. Although much has been learned about the molecular and cellular mechanisms of TBI in the past 20 years, these advances have failed to translate into a successful clinical trial, and thus there has been no significant improvement in treatment. Among the numerous barriers to finding effective interventions to improve outcomes after TBI, the heterogeneity of the injury and identification and classification of patients most likely to benefit from the treatment are considered some of the most significant challenges (Doppenberg et al., 2004; Marshall, 2000; Narayan et al., 2002).

The type of classification one develops depends on the available data and the purpose of the classification system. An etiological classification describes the factors to change in order to prevent the condition. A symptom classification describes the clinical manifestation of the problem to be solved. A prognostic classification describes the factors associated with outcome, and a pathoanatomic classification describes the abnormality to be targeted by the treatment. Most diseases were originally classified on the basis of the clinical picture using a symptom-based classification system. Beginning in the $18^{\text {th }}$ century, autopsies became more routine, and an increasing number of disease conditions were classified by their pathoanatomic lesions. With improvement of diagnostic tools, modern disease classification in most fields of medicine uses a mixture of anatomically, physiologically, metabolically, immunologically, and genetically defined parameters.

Currently, the primary selection criterion for inclusion in a TBI clinical trial is the Glasgow Coma Scale (GCS), a clinical scale that assesses the level of consciousness after TBI. Patients are typically divided into the broad categories of mild, moderate, and severe injury. While the GCS has proved to be extremely useful in the clini- cal management and prognosis of TBI, it does not provide specific information about the pathophysiologic mechanisms responsible for the neurological deficits. This is clearly demonstrated in Figure 1, in which all six patients are classified as having a severe TBI. Given the heterogeneity of the pathoanatomic features depicted in these computed tomography (CT) scans, it is difficult to see how a therapy targeted simply for severe TBI could effectively treat all of these different types of injury. Many tools such as CT scans and magnetic resonance imaging (MRI) already exist to help differentiate the multiple types of brain injury and variety of host factors and other confounders that might influence the yield of clinical trials. In addition, newer advances in neuroimaging, biomarkers, and bioinformatics may increase the effectiveness of clinical trials by helping to classify patients into groups most likely to benefit from specific treatments.

In order to review what is known about the heterogeneity of TBI and to develop strategies to capture and incorporate this information into research studies, the National Institute of Neurological Disorders and Stroke (NINDS) sponsored a workshop on Classification of TBI for Targeted Therapies in October, 2007. Co-sponsors included the Brain Injury Association of America, the Defense and Veterans Brain Injury Center, and the National Institute on Disability and Rehabilitation Research.

\section{WORKSHOP FORMAT}

The workshop organizing committee was co-chaired by Geoffrey Manley and Ramona Hicks, and members included Ronald Hayes, Linda Phillips, and Hilaire Thompson. Using the "grand challenge" approach for accelerating the advancement of science, three multidisciplinary teams were charged with proposing ways to (1) 


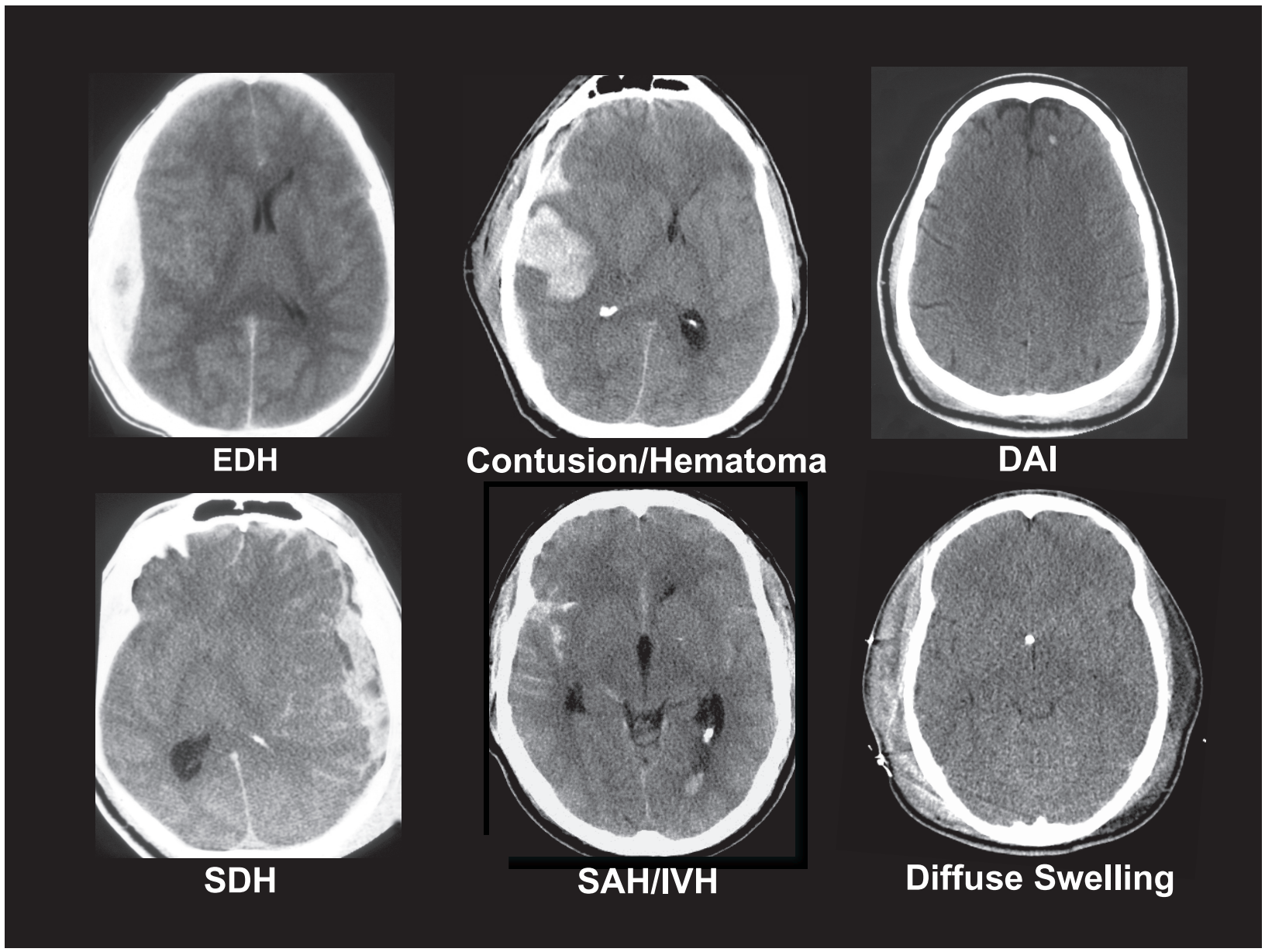

FIG. 1. Heterogeneity of severe traumatic brain injury (TBI). Computed tomography (CT) scans of six different patients with severe TBI, defined as a Glasgow Coma Scale score of $<8$, highlighting the significant heterogeneity of pathological findings. CT scans represent patients with epidural hematomas (EDH), contusions and parenchymal hematomas (Contusion/Hematoma), diffuse axonal injury (DAI), subdural hematoma (SDH), subarachnoid hemorrhage and intraventricular hemorrhage (SAH/IVH), and diffuse brain swelling (Diffuse Swelling).

use existing and emerging tools to detect the most common types and patterns of injury associated with TBI; and (2) develop a classification scheme that would cluster TBI patients into groups based on these major types of injury (see Appendix for workshop agenda). The teams had wide geographic representation across the USA and Europe, and represented scientists at various stages of their careers.

Prior to the workshop, each team was asked to prepare a position paper to:

- Summarize the current classification systems for TBI;

- List the most common forms of brain and neurovascular injury associated with TBI and their frequency;
- Identify currently available diagnostic tools and describe a protocol for using these tools to clearly delineate these common forms of brain and neurovascular injury and their severity;

- List data elements required for classifying TBI patients into groups based on the nature and severity of their injury;

- Describe the steps needed to develop a reliable, efficient and valid classification system for TBI that will be used to link specific patterns of brain and neurovascular injury with appropriate medical interventions.

The position papers were forwarded to an advisory panel before the workshop to allow them to prepare discussion questions. Information from the position papers 


\section{SAATMAN ET AL.}

and from the discussions during the workshop provided the basis for this manuscript.

\section{OVERVIEW OF CURRENT CLASSIFICATION SYSTEMS FOR TRAUMATIC BRAIN INJURY}

Head injuries most often have been classified by one of three main systems: (1) clinical indices of severity, used most often in clinical research to compare patients among centers; (2) pathoanatomic type, used most often to describe injuries for acute management; and (3) physical mechanism (i.e., causative forces associated with the injury), used most often in the biomechanics and prevention fields. In addition, pathophysiology of injury and evolution of injury cascades have been used to characterize aspects of TBI, particularly in basic science and research. Finally, multiple features can be combined, typically with the help of biostatisticians, to create prognostic classification criteria. Many of these schemes overlap with one another, and workers in different fields may use terminology in different ways, adding to some of the confusion in the head injury field and in clinical trial efforts. In addition, classification schemes will likely continue to evolve as new tools and concepts shed light on the causes and consequences of the disease process.

\section{Classification by Injury Severity}

In the head injury field, symptom classification generally has been based on clinical indices of injury severity at presentation. To date, the majority of clinical treatment trials for TBI have classified and entered patients based on neurologic injury severity criteria (Narayan et al., 2002). The 15-point GCS (Teasdale et al., 1974) is the most commonly used neurologic injury severity scale for adults, because of its high inter-observer reliability and generally good prognostic capabilities (Narayan et al., 2002). Patients with severe TBI, defined typically as GCS of 8 or less, have most often been enrolled in clinical trials. This group has the highest mortality and morbidity and was presumed to have the best chance of demonstrating a treatment effect. In addition, hundreds of preclinical trials, mostly using rodent models, have targeted animals with clinically and/or histologically significant injuries.

Other neurological severity scales include the Brussells Coma Grades, Grady Coma Grades, Innsbruck Coma Scale, and the FOUR score scale (Brihaye et al., 1978; Fleischer et al., 1976; Gerstenbrand et al., 1970; Wijdicks et al., 2005). A number of scales are also available to assess extracranial injury and physiologic instability which can influence outcome, including the Ab- breviated Injury Scale (AIS) (Medicine AftAoA., 1976; Medicine AftAoA., 1990) and the Injury Severity Score (ISS) (Baker et al., 1974). The AIS is a detailed injury scoring system for each of six body regions; the ISS is designed to quantify the severity of multiple body region injuries. The Trauma Score is a simplified scale which includes the GCS, respiratory rate, respiratory expansion, systolic blood pressure, and capillary refill in order to give an overall score (1-16) to assess injury severity (Champion et al., 1981). It has the advantage of being rapidly applied in the emergency setting.

Several problems arise from utilizing clinical injury severity indices as entry criteria into clinical trials for head injury. Factors such as patient age, extracranial injuries, and physiologic instability influence outcome (Marmarou et al., 2007; Murray et al., 2007). Thus, when trials are analyzed without taking these variables into account, confounding prognostic factors may override potential treatment effects. Furthermore, improvements in prehospital care and routine transfer of patients from community facilities to trauma centers mean that the majority of patients with more significant-appearing injuries now receive intervention prior to arrival at the study center. Intubation, sedation, pharmacologic paralysis, and intoxication complicate and often preclude the accurate assessment of neurologic injury severity on clinical grounds (Balestreri et al., 2004; Gabbe et al., 2003; Stocchetti et al., 2004). Specific populations of patients are difficult to assess with the GCS, including infants, young children and patients with pre-existing neurologic impairment. The GCS is also a poor discriminator for less severe TBI, which account for $80-90 \%$ of all cases.

A more fundamental issue surrounding trial entry solely based on clinical indices is whether this is really the most scientifically appropriate way to match specific treatments to specific patients. Clearly, patients who have in common a "severe" injury phenotype may vary widely in other injury classification schemes, such as those based on pathoanatomic or pathophysiological features (Fig. 1), which may be more relevant to the neuroprotectant action of a particular intervention.

\section{Pathoanatomic Classification}

A pathoanatomic classification describes the location or anatomical features of the abnormality to be targeted by a treatment, and generally falls into the scheme of "where and what" terminology. The majority of patients with more severe injuries have more than one injury type when classified in this way. Going from the outside of the head and working inwards, injury types include scalp laceration and contusion, skull fracture, epidural hemorrhage, subdural hemorrhage, subarachnoid hemorrhage 


\section{CLASSIFICATION OF TBI FOR TARGETED THERAPIES}

(SAH), brain contusion and laceration, intraparenchymal hemorrhage, intraventricular hemorrhage, and focal and diffuse patterns of axonal injury. Each of these entities can be further described by their extent, location, multiplicity, and distribution. Other radiologically and/or pathologically visible entities do not fit a strict "pathoanatomic" classification, but overlap with pathophysiologic classifications (e.g., ischemia, diffuse brain swelling) or mechanistic classifications (e.g., gunshot wounds, blast injuries).

That pathoanatomic type of injury influences outcome has long been recognized, particularly once imaging of patients with neurotrauma became routine (Gennarelli et al., 1982a). A number of classification schemes of these entities have been used for pathoanatomic description in many acute head injury studies, including the Marshall score for CT findings (Marshall et al., 1992) and the Rotterdam score (Maas et al., 2005). When applied to CT scans in early severe and moderate TBI, the Marshall score, an ordinal numbering score with 6 categories, has been shown to be powerful in predicting both the risk of increased intracranial pressure (ICP) and outcome in adults. The Marshall classification is widely used and pragmatic, but has many recognized and accepted limitations, including difficulties in classifying patients with multiple injury types and standardization of certain features of the CT scan. The Rotterdam score is a more recent and standardized CT-based classification system, which uses combinations of findings to predict outcome. This system has not been fully validated, and requires more study, but overcomes some of the limitations of the Marshall score.

\section{Classification by Physical Mechanism}

Etiological classification of head injuries by physical mechanism of injury has certain advantages in understanding how specific forces at specific magnitudes result in predictable patterns of injury. Thus, injuries can be classified according to whether the head is struck or strikes an object (contact or "impact" loading) and/or the brain moves within the skull (noncontact or "inertial" loading). The magnitude and direction of each type or combination of loading forces may predict type and severity of injury (Gennarelli et al., 1985). There is considerable, but not perfect, correlation between physical mechanism of injury and pathoanatomic injury type. For instance, most focal injuries, such as skull fracture, brain contusion, and epidural hematoma, result from impact loading, whereas inertial loading generally causes more diffuse injuries such as concussion, subdural hematoma and diffuse axonal injury (DAI). Recently there has been increased interest in blast mechanisms of brain injury, which are at present incompletely understood. Mechanistic classification has great utility in modeling injuries and in prevention. However, in clinical practice most often the loading conditions must be estimated from incomplete details of the traumatic event, and inferred in combination with the pathoanatomic findings and the clinical severity of injury.

\section{Classification by Pathophysiology}

Alternatively, pathophysiologic mechanisms may form the basis of an etiologic classification and/or characterization of targets for treatment. In head injury, these can include processes which are set in motion by the injury event and take time to evolve, as well as events which compound or complicate the brain injury such as systemic insults. One widely used scheme in head injury relating to pathophysiologic processes is that which differentiates "primary" versus "secondary" damage (Adams et al., 1994). While authors vary in exactly how these terms are used, in general, primary injury refers to the unavoidable, immediate parenchymal damage occurring at the time of injury, while secondary injury refers to potentially avoidable damage that occurs at variable times after injury. The importance of secondary insults, such as hypoxia, hypertension, hypercarbia, hyponatremia, and seizures, has gained widespread recognition. However, pathophysiologic classification schemes have not been commonly used in treatment trials. This may be due, in part, to challenges associated with capturing a spatiotemporal profile of the patient's injury, limited availability and usage of sophisticated monitoring techniques needed for measurement of physiologic parameters, and difficulties in distinguishing inevitable but progressive cell damage from potentially reversible injury cascades.

\section{Classification by Prognostic Modeling}

In early clinical research in head injury, investigators found that it was difficult to establish confident predictions of outcome after TBI on admission or to compare outcomes among centers. Thus, the GCS was designed as an early injury severity assessment tool and proved to have prognostic value (Jennett et al., 1975). However, the GCS provides information only in one knowledge domain (clinical severity) and may be difficult to measure on admission, as discussed earlier. Recent work from the International Mission for Prognosis and Clinical Trial (IMPACT) studies (Murray et al., 2007) has shown that predictions can be made on admission and has resulted in the development of three valid prognostic models of increasing complexity. This work is particularly relevant to mitigating the effects of prognostic variability in Phase III trials rather than specifically identifying subgroups of 
patients likely to benefit from a given targeted intervention.

In an ideal world, a TBI classification system would be able to select out patients with the potential to benefit from the intervention under investigation, from pathoanatomic, pathophysiologic, and prognostic perspectives. It is crucial to differentiate between the concepts of prognostic factors and factors which relate to a patient's potential to benefit from an intervention. Whereas certain prognostic factors are also markers of potential benefit (e.g., CT evidence of the nature of the brain injury), other factors such as age and impairment of consciousness are primarily prognostic factors. More direct, diagnostic measures of the underlying pathophysiology (e.g., ICP, lesion volume, microdialysis, blood flow, tissue oxygenation, coagulation status) are likely to have greater potential to identify patients who will benefit from a given intervention. Therefore, the intent and focus of this workshop was on developing an improved classification system for TBI that incorporates both diagnostic and prognostic perspectives with the goal of enhancing the success of future clinical trials.

\section{COMMON PATHOANATOMICAL AND PATHOPHYSIOLOGICAL SEQUELAE OF TRAUMATIC BRAIN INJURY}

Numerous postmortem studies and imaging studies have shown that there are four main pathoanatomical sequelae of TBI: contusions; SAH; hematomas, including epidural, subdural, and intraparenchymal lesions; and DAI (Fig. 2). While there is general consensus regarding the definition for most of these lesions, DAI is defined differently by different specialties, and the definition has evolved over time. Initially, the term was coined to describe the neuropathologic pattern created from pure inertial (noncontact) loading in a large animal model (Gennarelli et al., 1982b) which was developed to explain the clinical and pathologic findings seen in human patients with severe head injuries who died or had poor outcomes without mass lesions (Adams et al., 1977, 1982; Strich, 1956, 1961). Patients with this traditional definition of DAI are in profound coma from the onset of injury and usually have a poor outcome. As MRI techniques and sensitivity have improved, the radiologic pattern associated with DAI is now seen in patients with much milder injuries. Because some animal models and human patients have more restricted patterns of axonal injury than that seen in the classic descriptions, the term "traumatic axonal injury" (TAI) has been used for these more limited injuries. For these reasons, the term "DAI" needs to be carefully defined in clinical studies of TBI.

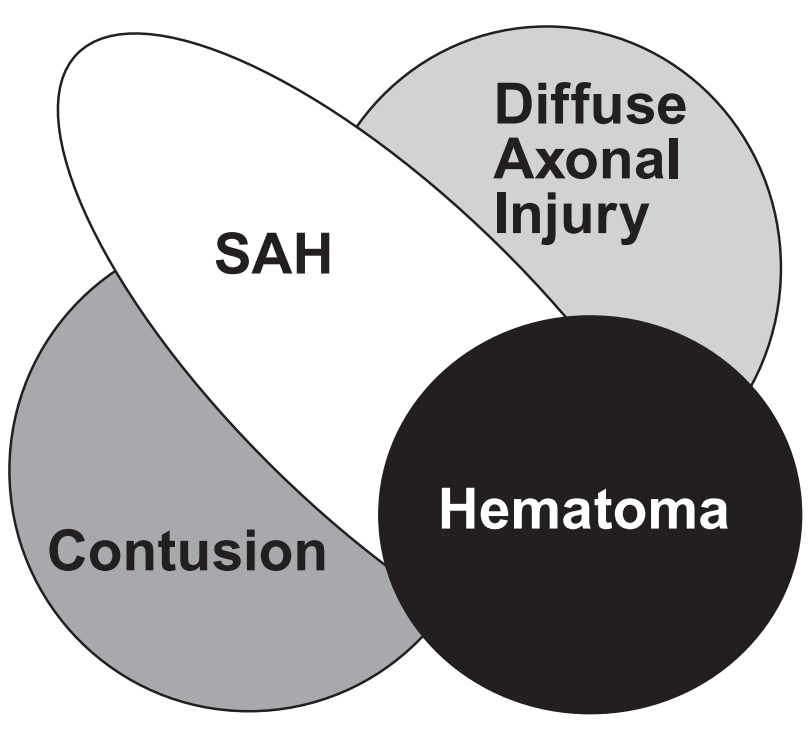

FIG. 2. Common pathoanatomic sequelae of traumatic brain injury (TBI). The Venn diagram represents the four main pathoanatomic sequelae of TBI: hematomas, including epidural, subdural, and parenchymal lesions; diffuse axonal injury; subarachnoid hemorrhage (SAH); and contusions.

Ischemic brain injury, cerebral edema and other pathophysiologic sequelae, in some cases, might be included in a "pathoanatomic" classification scheme, but in other instances would be more accurately described as pathophysiologic cascades or secondary insults. Depending on the specific entity and etiology, such processes could be viewed as therapeutic targets--that is, processes set in motion by the initial injury event which might be remediable. Alternatively, these could be viewed as confounding or prognostic variables which occur in some patients with a primary pathoanatomic injury type (for instance, subdural hematoma with an episode of delayed hypotension and ischemia). Many pathophysiologic sequelae have been extensively described in fatal TBI using autopsy specimens (Graham et al., 2005). Insights into the etiology and temporal evolution of these events, which may vary from patient to patient, have been provided by newer diagnostic modalities, such as CT angiography, cerebral blood flow measurement, transcranial Doppler (TCD), and angiography. These tools have also revealed additional pathophysiologic sequelae. For example, TCD data suggest that posttraumatic vasospasm may occur in up to $25 \%$ of patients with severe head injury (Oertel et al., 2005), often when severe basal SAH is present. Recent reports on blast injury suggest that vasospasm is especially important in modern military TBI (Armonda et al., 2006). Many of the above sequelae often coexist in patients with severe and fatal injuries as 


\section{CLASSIFICATION OF TBI FOR TARGETED THERAPIES}

well as patients with moderate and mild injuries as classified by GCS (Fig. 2).

Although the incidence of isolated, specific types of injury is known for fatal cases of TBI, the incidence, patterns and magnitude of overlapping injuries across a spectrum of injury severity have not been clearly described. To better understand these complex relationships, more information about common patterns of injury across the spectrum of TBI is critical for developing an improved classification system for targeted therapies.

\section{SUMMARY OF KEY WORKSHOP RECOMMENDATIONS}

The initial response of the workshop participants to developing a new classification system for TBI ranged from enthusiasm for improving the approach used for clinical trials by exploring the use of existing and new technologies to skepticism about what could realistically be achieved. Others believed that the workshop was "reinventing the wheel" because similar discussions had taken place decades ago. In retrospect, the varying perspectives in terms of enthusiasm for the concept, scientific and medical disciplines, years in the field, and geographic localities all contributed greatly to the workshop discussions and recommendations. In the end, there was widespread agreement that patient selection based on the pathoanatomic features of the individual's brain injury should be the cornerstone for a new TBI classification approach for clinical trials.

A central tenet of this approach is that brain injuries with similar pathoanatomic features are likely to share common pathophysiologic mechanisms of cell and tissue injury. In this manner, patients with the greatest potential to benefit from a given intervention, based on the mechanisms of action of the therapy, would be selected for study. The number of nonresponders should also be reduced, thereby increasing effect size.

The conceptual framework of a multidimensional, pathoanatomic classification approach for clinical trials is as follows:

- Use preclinical models to: (1) Evaluate pathophysiologic mechanisms and identify those pathoanatomic types of TBI for which the mechanisms are relevant. This would establish the "targeted injury type." Although certain brain injury pathologies such as diffuse brain swelling, hypoxia, and ischemia do not fit cleanly into pathoanatomic classification categories, mechanisms underlying these TBI-associated pathologies also represent important targets for study. (2) Establish that an intervention strategy de- signed to affect one or more of these mechanisms mitigates cellular damage and functional impairment in the relevant targeted injury type(s). (3) Guide selection of patient populations likely to benefit (i.e., those with the targeted injury type).

- Determine which patients have the targeted injury type(s) using an initial diagnostic entry criterion (e.g., based on CT or MRI).

- Measure or grade injury severity, and include or exclude patients according to predetermined selection parameters. The approach for assessment of severity may vary with injury type but would ideally describe distribution (e.g., extent and location of injury measured using a radiologic grading scheme), clinical effects (e.g., GCS and neurologic exam) and possibly physiologic effects (e.g., microdialysis, biomarkers).

- Identify and characterize additional brain injury types that may be present because these may influence outcome.

- Use multivariate prognostic modeling to further refine inclusion/exclusion criteria and to permit stratification. These models can incorporate varying degrees of complexity, as desired, including demographic data (e.g., age, gender, education, cause of injury, ethnicity, genotype), physiological data (e.g., extracranial injuries, hypoxia, hypo/hypertension, temperature, elevated ICP, apnea, acidosis, cerebral blood flow, biomarkers), and data related to clinical status (e.g., GCS, level of alertness, pupillary status, neurologic exam, neuropsychologic exam). The inclusion of GCS and an assessment of extracranial injuries (e.g., ISS/AIS) was felt to be of particular importance.

- Select endpoints for outcome assessment which are relevant to the targeted injury type and utilize tools optimal for detection of the targeted pathophysiology. Outcomes may include radiologic endpoints (e.g., MRI, MR spectroscopy, diffusion tensor imaging (DTI), CT perfusion), physiologic endpoints (e.g., microdialysis, electroencephalogram (EEG), ICP, biomarkers, brain $\mathrm{pO}_{2}$, cerebral blood flow), or clinical endpoints (e.g., Glasgow Outcome Scale (GOS), health-related quality of life measures, focal neurologic deficits, neuropsychologic exam). The times at which endpoints are measured may vary with scientific question.

The workshop participants acknowledged that there are substantial hurdles to overcome before implementation of a new classification system could take place. However, an atmosphere of optimism persisted, based in large part on the firm belief that many of the tools needed to 


\section{SAATMAN ET AL.}

implement such a system are already in hand. We have learned a great deal about injury mechanisms and the primary pathologies unique to various subtypes of brain injuries through animal and human studies. Our clinical assessment tools are useful and, in many cases, are widely available and well validated. We have learned important lessons from previous clinical trials (Narayan et al., 2002), and multivariate prognostic models have been developed (Murray et al., 2007). Nonetheless, there were several recommendations regarding the refinement of tools used for the assessment of TBI and for data collection and dissemination:

- Establish a patient database to: (1) Characterize common pathoanatomic patterns of injury across the entire spectrum of injury severities and across the lifespan. (2) Identify correlations between demographics, injury severity, physical mechanisms and pathoanatomic patterns of injury to enable rapid diagnosis and treatment of common patterns of injury. (3) Share clinical data across TBI research centers for the purpose of developing a pathoanatomic classification system.

- Trial inclusion criteria should be broadened to include less severely injured TBI patients. Many expressed concern that patients classified as having mild TBI based solely on GCS are excluded from clinical trials, despite debilitating and persistent symptoms.

- Neuroimaging modalities such as CT are likely to be used as the primary tool to identify the features of a TBI, thereby enabling pathoanatomic classification. Existing CT grading schemes are useful; however, they should be modified to include additional detail, such as information on lesion location, and further validated.

- More widespread use of acute MRI will be important to provide additional detail necessary for accurate pathoanatomic classification, particularly of the TAI/DAI spectrum. Efforts should be coordinated to identify and eliminate barriers to the implementation of acute MRI for TBI clinical trials and to standardize and validate MRI grading schemes.

- Incorporating endpoints related to the specific, targeted pathophysiology will be especially critical for evaluating the success of Phase II trials, and should increase sensitivity of effect detection.

- Additional functional outcome measures that simulate real-life tasks or functions with ecological validity should be developed, validated, and incorporated in a standardized fashion into future clinical trials.

- More complex statistical and bioinformatics techniques (e.g., covariate adjustment, ordinal outcome modeling) are necessary to increase sensitivity of trials and allow classification utilizing multiple vectors.

- Additional education/instruction is needed to improve standardization and reliability in the use of existing tools, such as grading and classification schemes.

- A mutually agreed upon set of common data elements for TBI for all levels of severity should be established, in cooperation with NINDS initiative on common data elements (www.nindscommondataelements.org/CommonForms.aspx).

- Appropriate elements and tools for special populations (e.g., pediatric TBI, geriatric TBI, blast TBI) should be developed.

- Possibilities should be explored for expanding the Traumatic Coma Data Bank or establishing a new databank as a basis for developing a classification system for targeted therapies. In addition, state-ofthe-science platforms for data sharing and analysis should be explored.

\section{ROUNDTABLE DISCUSSIONS}

\section{Roundtable Discussion: Pathoanatomic Heterogeneity and Laboratory Models}

This roundtable group focused on the heterogeneity of TBI pathology and its implications for animal models and preclinical studies. Goals for the discussion were to (1) identify in vivo, in vitro, and computational models that might be useful in the validation of tools that detect and discriminate between the various types of TBI, (2) compare the advantages and disadvantages of these models for this purpose, and (3) recommend ways to address any limitations and gaps in the models.

Preclinical animal models of focal and diffuse insults (Povlishock et al., 1994; Thompson et al., 2005) were discussed. Traditionally, focal insult models have emphasized production of targeted contusion and local ischemia, whereas the most commonly used diffuse injury models aim to create DAI or diffuse ischemia. Contusion can be produced by controlled cortical impact and by the generation of either local hematoma or hemorrhagic lesions. Focal ischemia is commonly produced with controlled vascular occlusion. Models producing inertial acceleration-deceleration or acceleration through a distributed impact are typically used for generating DAI. Manipulations of cerebral perfusion and oxygenation to generate diffuse ischemia may be useful to differentiate neuropathologic profiles of global ischemia from DAI (Povlishock et al., 2005) aiding in the design of targeted therapies. Other variants of head injury, including pene- 


\section{CLASSIFICATION OF TBI FOR TARGETED THERAPIES}

trating missile and blast injury, are of increased importance in the military arena (Warden, 2006). The development and evaluation of models for these other forms of TBI are challenged by the paucity of information regarding their pathobiology and the fact that recovery assessment may be seriously confounded by complex posttraumatic stress disorder (Kennedy et al., 2007; Kim et al., 2007).

While in vitro TBI models provide elegant ways to test detailed aspects of trauma pathophysiology, they generally have not been exploited for discriminating between the different types of TBI for the purposes of clinical intervention. Computational models of relationships between applied physical forces and tissue damage have been utilized primarily for injury prevention research rather than for guiding treatment strategies. However, prediction of anatomical location of injury, vulnerable tissue or cell types, or the severity or progression of a lesion could have important applications to a pathoanatomic classification system and may represent an opportunity for the future.

In summary, in vivo modeling will continue to provide critical insights into the pathobiology of specific types of TBI and the efficacy of candidate therapeutics. Although a sound roster of animal models exists for evaluating major brain injury types, the roundtable participants acknowledged certain limitations and made the following recommendations:

- Thorough pharmacokinetic studies of investigational drugs should be done in a standardized, well-controlled fashion.

- Rodent models predominate in the field of TBI, leading to challenges with respect to scale and anatomy when translating to humans. Differences in brain size, organization and maturation may affect, for example, the biomechanical response of the brain, the delivery of a therapeutic, and the functional or physiologic consequences of "comparable" injuries across species. Therefore, a significant change in large areas of the lissencephalic rodent brain may only translate into small effects in the gyrencephalic human, or vice versa (Manley et al., 2006; Povlishock et al., 1995; Statler et al., 2001). The group recommended moving some of the injury paradigms into larger, gyrencephalic species. This was felt to be particularly important for validating imaging modalities through postmortem assessment of brain pathology.

- The majority of preclinical studies have focused on acute interventions and outcomes. In contrast, the success of human trials has been judged using longterm outcome, assessed months after TBI. The chronic posttraumatic interval is characterized by the activation of a secondary adaptive recovery, which is not generally considered in acute or subacute animal assessments. Preclinical model analysis should be extended to include periods of recovery and brain plasticity. In addition to the assessment of motor and sensory recovery, other tests should be developed to dissect complex brain recovery mechanisms (e.g., cognitive integration, emotion) which occur with long-term survival.

- Variability in pre-existing physiological status and in extracranial injuries and complications resulting from traumatic injury in humans is not represented in animal models. Real world patient heterogeneity requires a variety of interventions applied across the phases of injury and recovery. Well-monitored animals subjected to standardized models of TBI have less variability and, therefore, a much lower risk-tobenefit ratio for a given treatment. The group recommended that laboratory modeling of TBI should always consider the 'bedside to bench' strategy, using human brain pathology to identify the specific biological variables most affected by injury. Such variables should exhibit the most significant effects in animal models and better replicate the human condition. In addition, the fact that controls for systemic effects associated with TBI are not in place for humans should be considered when translating the efficacy of interventions from rodent models. Given that we have good rodent models in place, choosing therapeutic manipulations which result in larger, measurable signals may increase the likelihood that experimental treatment effects will be detectable above the expected variability of outcomes in humans.

- Models do not capture the full spectrum of injury severity. This is especially true for milder forms of injury, for which new models should be validated and fully characterized.

- Models of blast injury need further development and validation for studies on both military and civilian populations.

- Animal models of posttraumatic stress should be developed and tested for interaction with TBI. This combination is emerging more frequently, particularly in association with blast injury.

\section{Roundtable Discussion: Acute Clinical Monitoring}

The goals of this roundtable discussion were to compare the advantages and disadvantages of acute clinical monitoring tools, to make recommendations for which tools to use and when, and to recommend ways to ad- 


\section{SAATMAN ET AL.}

dress limitations in the current tools. By tools, here, we refer to indices of patient condition that might be used for management decisions, severity assessment, or surrogate outcome measures in some clinical trials. These tools might include various ways to more fully characterize (a) specific pathoanatomic type or extent of injury, (b) severity of neurologic or neuropsychologic deficits, (c) pathophysiology of injury-initiated cascades or secondary insults, or (d) evolution of injury. In clinical trials, each of these features might be used to standardize entry criteria or to follow a specific target for intervention.

The primary initial evaluation of a patient with a TBI currently includes the GCS, complemented by CT and a detailed neurologic exam to the extent feasible and dictated by patient clinical status. The initial clinical evaluation, however, is frequently unreliable and further clouded by use of sedative and neuromuscular blockade medications in the emergency setting. These difficulties with the early clinical evaluation run counter to the need for rapid assessment, recruitment, and enrollment of patients in clinical trials addressing early therapeutic interventions. Therefore, additional concrete and valid assessments tools are needed in the early phase.

- In the acute phase ( $0-4 \mathrm{~h})$, there is consensus that despite the limitations noted, the GCS score remains the standard and most well-validated index of overall neurologic injury severity. However, it is most helpful on the more severe end of the injury spectrum. Protocols are clearly needed to better characterize injury type and severity in patients on the less severe end of the spectrum. This may be in the form of early MRI, serum biomarkers, rapid neuropsychologic tests, or innovative techniques such as magnetoencephalography attempting to quantify the extent of neurologic/physiologic disturbance as a result of a more minor or moderate TBI.

- Moving beyond the acute phase evaluation and into the "Intensive Care Unit phase" (approximately 4$12 \mathrm{~h}$ postinjury), additional parameters may be incorporated into the early clinical evaluation and characterization of injury. Appropriate tools might serve to better define specific injury type, extent, pathophysiology, and evolution of injury over time. Where appropriate, ICP monitoring, microdialysis sampling, brain tissue $\mathrm{pO}_{2}$ measurements, and EEG may have a role.

- Age-appropriate measurement techniques are needed to distinguish specific injury types, pathophysiology, and evolution of injury in pediatric patients, the aged, or other populations in which these features may be distinctive.
- More widespread use and validation of existing tools, such as CT perfusion scans, electrophysiology, and DTI, is clearly needed. Additional functional and feasible acute measures (e.g., biomarkers, CT perfusion, early neuropsychologic batteries) must be developed, validated, and related to delayed modalities with known prognostic significance.

- Our current armamentarium is insufficient, and there is a need for better, more sophisticated tools to measure extent of injury and occurrence of specific pathophysiologic mechanisms as summarized in Table 1.

- In order to reduce multicenter variance, clinical monitoring in the acute phase should be performed with uniformity across participating centers and in such a manner that common data elements are populated and sample sizes for clinical studies controlled.

\section{Roundtable Discussion: Neuroimaging}

The goals of this roundtable discussion were to compare the advantages and disadvantages of neuroimaging tools, to make recommendations for which tools to use and when, and recommend ways to address limitations and gaps in the current tools. The discussion began by acknowledging the distinction between the use of imaging for acute classification of pathoanatomic injury type and the use of neuroimaging tools to answer specific research questions about pathophysiology, extent of injury, secondary injury, evolution of pathology, and treatment effects.

With respect to use of imaging for acute classification, it is widely accepted that for most injury types, especially at higher levels of injury severity, CT scan is the initial test of choice (Table 2). This arises from its wide availability and its high reliability in identifying the presence of hemorrhages, contusions, and mass effect, which guides acute management and has proven prognostic significance. However, more complex classification schemes, such as the Rotterdam scale and others, are needed to handle heterogeneity in the size, location, and multiplicity of lesions, as well as findings such as swelling, loss of grey-white differentiation, presence or absence of cisterns and sulci, tissue shifts, and herniation effects. Thus, it was felt that within most of the common pathoanatomic categories, data are available from tools already at hand to help stratify patients and predict outcome using current neuroimaging techniques. It was also recognized that development of computer-aided diagnostic tools is needed for more objective and quantitative image analysis and to improve workflow for clinical trials.

For some diagnoses, MRI has higher sensitivity and specificity for detection and classification, particularly 


\section{CLASSIFICATION OF TBI FOR TARGETED THERAPIES}

Table 1. Tools for Pathoanatomic and Pathophysiologic Sequelae

Common pathoanatomic

Acute monitoring and diagnostic tools

and pathophysiologic sequelae

Available Additional validation needed

Hematoma, contusion, subarachnoid hemorrhage

Imaging, ${ }^{\mathrm{a}}$ clinical exam

Biomarkers of coagulopathy, assessment of ischemia/perfusion (Xenon CT, CT perfusion), risk for hemorrhage expansion, risk for malignant intracranial hypertension

Diffuse axonal injury

Intracranial hypertension

Cerebral hypoxia/ischemia ${ }^{b}$

Cerebral swelling ${ }^{\mathrm{c}}$
Imaging, ${ }^{\mathrm{a}}$ clinical exam

ICP monitoring, clinical exam PET, clinical exam, Xenon CT

Imaging, ${ }^{\mathrm{a}}$ clinical exam
Biomarkers, imaging (susceptibility weighted imaging, diffusion tensor imaging)

Non-invasive ICP monitoring

Continuous bedside measurements $\left(\mathrm{PbtO}_{2}, \mathrm{SjvO}_{2}\right.$, near-infrared Spectroscopy, TCD), microdialysis Continuous bedside measurements (CBF, brain compliance, tissue water content)

\section{${ }^{\text {aSee Table } 2 .}$}

bIncludes post-traumatic vasospasm.

${ }^{\mathrm{c}}$ Cerebral swelling due to increased blood volume or edema.

$\mathrm{CBF}$, cerebral blood flow; CT, computed tomography; ICP, intracranial pressure; PET, positron emission tomography; $\mathrm{PbtO}_{2}$, brain tissue oxygen tension; $\mathrm{SjVO}_{2}$, jugular venous oxygen tension; TCD, transcranial Doppler.

Table 2. Imaging Modalities for Pathoanatomic and Pathophysiologic Sequelae

\begin{tabular}{|c|c|c|c|c|c|c|}
\hline Modality & Hematoma & Contusion & $S A H$ & $D A I$ & Ischemia & Vasospasm \\
\hline $\mathrm{CT}$ & +++ & ++ & +++ & $+/ 0$ & $+/ 0$ & 0 \\
\hline CTA & 0 & 0 & + & 0 & + & +++ \\
\hline СTP & + & + & 0 & 0 & ++ & ++ \\
\hline T2 FLAIR & + & +++ & +++ & + & + & 0 \\
\hline 1.5T T2* GRE & + & + & +++ & + & 0 & 0 \\
\hline 3T T2* GRE & + & + & +++ & ++ & 0 & 0 \\
\hline SWI & +++ & ++ & ++ & +++ & 0 & 0 \\
\hline DTI & + & + & 0 & +++ & +++ & 0 \\
\hline PWI & + & + & 0 & + & ++ & ++ \\
\hline MRSI & 0 & 0 & 0 & ++ & + & 0 \\
\hline MRA & 0 & 0 & + & 0 & ++ & +++ \\
\hline fMRI & 0 & 0 & 0 & $?$ & 0 & 0 \\
\hline PET & 0 & + & 0 & $?$ & +++ & 0 \\
\hline
\end{tabular}

Scoring: little/no data, ?; insensitive, 0 ; minimally sensitive, $+/ 0$; mildly sensitive, + ; moderately sensitive ++ ; highly sensitive, +++ .

CT, computed tomography; CTA, computed tomography angiography; CTP, computed tomography perfusion; DAI, diffuse axonal injury; DTI, diffusion tensor imaging; fMRI, blood oxygenation level dependent (BOLD) functional magnetic resonance imaging; MRA, 3D time-of-flight MR angiography; MRSI, MR spectroscopic imaging; PET, positron emission tomography; PWI, dynamic susceptibility contrast perfusion-weighted imaging; SAH, subarachnoid hemorrhage; SWI, susceptibility-weighted imaging; T2 FLAIR, T2-weighted fluid attenuated inversion recovery; T2*GRE, T2*-weighted gradient echo. 


\section{SAATMAN ET AL.}

for DAI and more subtle imaging manifestations found in "concussive" type head injury. Therefore, for purposes of some study questions, MRI would be a preferable initial imaging modality. In the spectrum of diffuse white matter injuries, fluid-attenuated inversion recovery (FLAIR) and T2 sequences are sensitive for non-hemorrhagic white matter lesions, and gradient echo and susceptibility-weighted imaging for blood products. Diffusion-weighted imaging is also very sensitive for DAI but, as is the case with ischemia, its sensitivity is limited to the acute setting. It is not yet clear whether DTI or other emerging techniques will demonstrate even greater sensitivity in recognizing axonal injury or will be able to track or predict functional recovery or response to intervention, though significant progress has been made (Gallagher et al., 2007).

Both MRI and specialized CT techniques provide unique information about some pathophysiologic processes involving ischemia and blood flow abnormalities (Table 2). These techniques include CT and MR perfusion, diffusion-weighted imaging, and CT and MR angiography. With some MR techniques, the presence of blood products creates artifacts which can distort the images, making these applications less robust in the setting of TBI than they have proven to date in nontraumatic ischemic pathologies such as stroke. Currently, perfusionCT techniques do not offer whole-brain coverage, a drawback that will be eliminated with the advent of large coverage (256- or 320-slice) CT scanners. Isotope or metabolite-based techniques such as xenon CT, single photon emission CT or positron emission tomography (PET) are useful at present for patients with TBI mostly in the research setting, but have given insight into the complex relationship between brain perfusion and metabolism While neuroimaging techniques have the significant disadvantage of reflecting only one point in time, imaging has the major advantage of providing regionspecific information. In contrast, the majority of bedside cerebral monitoring techniques concerned with cerebral blood flow, oxygen tension, and other physiologic variables can provide continuous measurements, but they reflect only one geographic point in a very large and often heterogeneous intracranial compartment. For this reason, it was felt that neuroimaging would continue to play an increasing role in research questions involving cerebral hemodynamics and metabolism.

Other neuroimaging techniques may be useful for tracking specific aspects of pathophysiology or recovery. MRI volumetric analyses have been used by several groups to follow head-injured patients over time and to correlate patterns and extent of tissue loss in specific regions with neuropsychologic outcomes. MR spectroscopy has been used in severe injuries to show major loss of metabolic integrity, but at present is less well studied for distinguishing among patients or predicting outcome in those with milder injuries. There has been some hope that specialized MR spectroscopic or PET techniques which can localize and quantify neurotransmitters or other molecules may prove useful in tracking response to treatment with various neuroprotective or psychoactive medications. Although functional MRI has been used to track recovery in some acquired conditions such as surgery for tumors or epilepsy, its use in trauma depends in part on the location of the damage and specific functions impaired. Magnetoencephalography, used largely in studying epilepsy, is still in the early stages of use in head-injured patients, and as yet little is known about what information it may provide in this setting. Most of these advanced imaging techniques currently suffer from the lack of normative databases and protocol standardization.

Overall, the imaging roundtable group concluded that current imaging techniques provide an excellent start on injury classification, especially with schemes which recognize the varying severity and multiplicity of injuries. In addition, the wide variety and continuous refinement of emerging imaging techniques should be watched closely and kept in mind as TBI research moves ahead. These techniques will become increasingly important for improved injury classification, pathophysiology characterization, prognostication, and treatment effects analysis.

\section{Roundtable Discussion: Biomarkers}

The goals of this roundtable discussion were to evaluate the status of biomarkers as a tool for TBI classification and to recommend ways to facilitate research in this area. A number of putative serum, cerebrospinal fluid (CSF), or microdialysate biomarkers have been evaluated in animal models and clinical studies of TBI, with S100 and neuron-specific enolase being among the most widely investigated. However, many of these candidate biomarkers have failed to exhibit adequate sensitivity and specificity for central nervous system (CNS) injury or yield significant prognostic value. Therefore, the discussion of a role for biomarkers in clinical trials for TBI was infused with an air of caution. As with any newly developed tool, biomarkers should demonstrate diagnostic or prognostic value above that available with existing tools. In general, biomarkers were felt to be insufficiently characterized to serve as a classification tool, a prognostic factor, or a surrogate outcome marker in clinical trials in the immediate future. However, there was enthusiasm for the continued development and validation of biomarkers. 


\section{CLASSIFICATION OF TBI FOR TARGETED THERAPIES}

The development of biomarkers generally progresses from detection and characterization in the brain to measurement and validation in CSF and then in serum. The roundtable group discussed a number of challenges that arise during this process, and the implications for the use of biomarkers in TBI clinical trials. With respect to a potential pathoanatomic classification approach for TBI patients, the most important question is whether current biomarkers are useful for differentiating injury type. That is, do we have biomarkers specific for contusion versus subdural hematoma? Workshop participants agreed that we do not currently have this capability and felt this represented an important, but long-term, goal of biomarker development.

In the initial classification of injury, biomarkers might be used in an alternative fashion, to grade injury severity. Levels of certain biomarkers may correlate with injury severity, as assessed by other clinical indicators such as GCS (Pineda et al., 2007). The use of a biomarker to assess injury severity could avoid problems with unreliable GCS assessments in patients who are intoxicated or intubated. While CSF biomarkers that reflect injury severity might be available for widespread use in the near future, more skepticism was expressed regarding the likelihood that serum biomarkers would possess adequate sensitivity to differentiate injury severity. Additionally, the loss of spatial information in CSF or serum biomarker data raised concerns about the ability of biomarkers to differentiate between a mild injury encompassing a large brain area and a severe injury involving a small region of brain, for example, or between two equivalent-size lesions in different parts of the brain that result in greatly different clinical presentations. Local biomarker levels assessed through microdialysis may provide critical information in these types of cases. Nonetheless, biomarkers likely represent a tool which will supplement, rather than replace, existing approaches such as neuroimaging and GCS in classification of patients for trials.

In addition to aiding in the initial assessment of injury type and severity, biomarkers may serve as a prognostic indicator for TBI. The utility of biomarkers in improving prognostic capabilities lies in the sensitivity and specificity of the biomarker. There was particular enthusiasm for the development of biomarkers that would aid in the prognosis of mild TBI. In a patient with a normal CT scan or MRI, a biomarker that could predict worsening neurological status would have great clinical utility. Similarly, biomarkers that could predict the likelihood of secondary injuries such as ischemia or hypoxia would be valuable.

In summary, the roundtable participants encouraged the continued exploration and validation of biomarkers for TBI. Biomarkers will likely supplement existing tools such as GCS and neuroimaging for the initial classification of brain injury in the near future. The use of biomarkers to identify at-risk patients with mild TBI or to differentiate injury pathology types were felt to be important, but long-range, goals.

\section{Roundtable Discussion: Clinical Outcome Measures}

The goal of this roundtable discussion was to describe the role of outcome measurements in developing a pathoanatomic classification system for targeted therapies. To this end it was agreed that the choice of an outcome measure determines the degree to which the efficacy of any clinical trial is, at minimum, documented and, at maximum, comprehensively defined. This is relevant to the issue of classification, because with greater clarity in defining the focus of the intervention, ramifications for outcome measurement may emerge. For example, does greater specificity in defining the type of brain injury or the nature, etiology and type of neuropathology lead to different expectations with respect to outcome and, therefore, different measurement needs?

A clear view of the range of points at which "success" can be measured is needed prior to choosing one or more appropriate measures to describe the impact of an intervention. For example, the impact of an intervention designed to reduce mortality or secondary complications during the acute stages of care may require assessing both short-term and long-term functioning within different International Classification of Functioning domains (World Health Organization, 2001). This intervention will require a different type of outcome assessment than one that is seeking solely to improve functioning in the community. Thus, the GOS (Jennett et al., 1975, 1981) or Disability Rating Scale (Rappaport et al., 1982) might be appropriate for use as global measures of immediate outcome in an acute trial. In contrast, measures that assess in more detail constructs such as participation and healthrelated quality of life, for example, the Mayo-Portland Adaptability Inventory (Lezak et al., 2003), the Neurobehavioral Rating Scale (Levin et al., 1987), the Participation Objective, Participation Subjective (Brown et al., 2004), or the Satisfaction with Life Scale (Diener et al., 1985), might be useful when examining the more distal impact of residual impairments or disability on the person's functioning within activity and participation domains.

Consideration must be given to the appropriate level of outcome analysis when examining specific questions with regard to the effectiveness of an intervention. For example, while neuropsychological tests are sometimes viewed as useful outcome measures for clinical trials, the utility of test scores in defining outcomes depends on the 


\section{SAATMAN ET AL.}

purpose of the trial. Thus, if a pharmaceutical is hypothesized to improve memory function, it is appropriate to use a neuropsychological test of memory function to document outcome. However, neuropsychological tests have been criticized for their lack of "ecological validity" (Burgess et al., 2006); that is, performance on a test often bears little relationship to the person's day-to-day function. In response, Alderman et al. (2003) have developed the "Multiple Errands Test" to describe the level of executive deficits of individuals with brain injury in the context of carrying out everyday tasks. This test examines the performance of the individual in standardized situations (e.g., purchase of a greeting card, locating a business in a building) and has been found to be sensitive in discriminating between different types of deficits in executive function. Further research examining the validity of this approach is needed prior to recommending that it be applied as an outcome measure in a clinical trial. Another alternative to neuropsychological tests is the Assessment of Motor and Process Skills (AMPS) (Merritt et al., 2003). This measure must be administered by an AMPS-certified occupational therapist and consists of groups of functional tasks of graded difficulty, for example, cooking and other homemaking activities. It has been found to be moderately correlated with cognitive function (Bouwens et al., 2008) and to be sensitive to the effects of rehabilitation (Waehrens et al., 2007). Thus, while the AMPS has promise as an outcome measure, its widespread application is limited by restrictions placed on its use. Considerable development research is needed before ecologically valid measures are appropriate for consideration as outcome measures in clinical trials.

\section{Roundtable Discussion: Data Collection and Management}

The goals of this roundtable discussion group were to identify and discuss available databases and to make recommendations for which, if any, of these resources might be useful for developing a pathoanatomic classification system for TBI. While a well-designed and well-conducted (RCT) remains the gold standard, data analyses of disease registries and cohorts have contributed significantly to the development of current guidelines for the management of severe TBI (Guidelines, 2007) and to the formulation of research hypotheses. Historically, two databases have made major contributions to the current management of TBI. The CNS Trauma Database included all severities of head injury and preceded the Traumatic Coma Data Bank, which was primarily limited to severe brain injuries. Recently, the IMPACT team in Europe created a large database by combining information from 9205 patients collected in eight RCTs and three observational studies (Marmarou et al., 2007). However, the
IMPACT database includes data from nearly 20 years ago, which do not necessarily reflect current relationships between classifiers and outcome. In addition, as with many databases, datasets were not collected in a uniform manner, leading to large amounts of missing data for some items (Van Beek et al., 2007).

Thus, current databases are not optimal for achieving the purpose described by this workshop, which is to develop a classification system by which to better separate patients who will respond to a treatment from those who will not. While one of the underlying tenets of a RCT is the presumption of clinical equipoise, the notion of performing a trial amongst those who have a good chance of responding to a particular form of treatment is very generally accepted (Friedman et al., 1998). The problem this sets for classification is that each treatment may require a different classifier; alternatively, the classifier may be able to finely divide the patients into small subgroups which are then collapsed into treatment-specific groups. Regardless, the classifier must be designed to fit a specific purpose.

Considerations and suggestions for data collection and management follow.

- A new database should be created with uniform data collection criteria on a well-defined set of possible classifiers. This dataset could then be used to validate current classifiers and create new classifiers.

- One form of this database may grow from the NINDS effort to define a common set of data elements (www.nindscommondataelements.org/CommonForms.aspx) with additional TBI-specific elements added using a critical evaluation of existing core datasets and the recently established BrainIT group (Chambers et al., 2006; Piper et al., 2003). If these items were to be collected from every future clinical trial in TBI and placed in a common data repository, a contemporaneous equivalent to the IMPACT database could be created. The multisite phase III proTECT trial could serve as a model to begin such data collection.

- Common data elements for inclusion in a TBI database may be arranged into a manageable number of modules: demographic, physiologic, clinical, and imaging. Much of the groundwork in this area has been begun by the IMPACT team and should be included in the design of a new database. Each data element could be further grouped as a classifier based on whether it is experimental or experiential (Table 3).

- Any newly created database would need to be open to the entire TBI research community. 
CLASSIFICATION OF TBI FOR TARGETED THERAPIES

Table 3. Common Data Elements for Inclusion in a TBI Database

\begin{tabular}{lcc}
\hline & \multicolumn{2}{c}{ Status of classifier } \\
\cline { 2 - 3 } Common data cluster & Evidence-based & Experimental \\
\hline Demographic & $\begin{array}{c}\text { Age, education, cause of } \\
\text { injury (e.g., MVC, fall) }\end{array}$ & Gender, genotype \\
GCS, systemic injuries, & Inflammation, oxidative stress \\
ethanol, serum glucose & Biomarkers, microdialysis \\
Physiologic & MAP, CBF, PbtO, SjvO & \\
& neurophysiologic testing & \\
Imaging & Extra-axial hematoma, intra- & Perfusion MRI, DTI, NMR \\
& axial hematoma, DAI, & spectroscopy, PET \\
& fracture & \\
\hline
\end{tabular}

CBF, cerebral blood flow; CPP, cerebral perfusion pressure; DAI, diffuse axonal injury; DTI, diffusion tensor imaging; GCS, Glasgow Coma Scale; ICP, intracranial pressure; MAP, mean arterial pressure; MR, magnetic resonance; MRI, magnetic resonance imaging; MVC, motor vehicle crash; $\mathrm{NMR}$, nuclear magnetic resonance; $\mathrm{PbtO}_{2}$, brain tissue oxygen tension; PET, positron emission tomography; $\mathrm{SjVO}_{2}$, jugular venous oxygen tension; $\mathrm{TBI}$, traumatic brain injury.

- Any new database must carefully define the reference population, which will define the inclusion and exclusion criteria and the population to which the information derived is to be generalized. For example, if the database is to include all severities of brain injury, are the data items which apply to a patient with a GCS of 4 likely to be of interest in a patient with a GCS of 15 ? These definitions will then determine the number and kind of clinical sites from which to collect information. Even if a perfect set of data items is selected at a given time, it is likely that this set will need modification as knowledge grows.

- The manner in which data are collected is also a substantial issue for the utility of such databases. Collecting data without a clear reason is difficult, as witnessed by the amount of missing data in existing databases. Any new database will require using all possible technical means to automate data collection to reduce the burden on the individual sites.
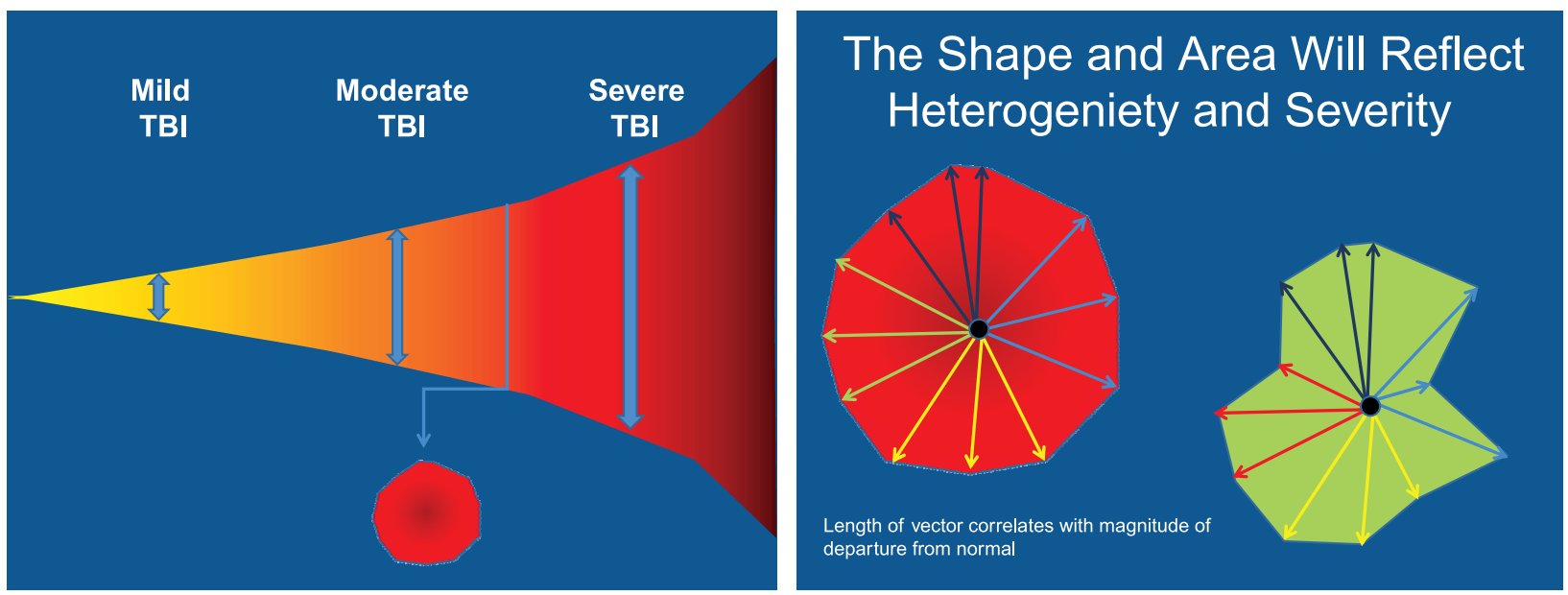

FIG. 3. Multiple vector-based analytical scheme. Tensor representations of the high dimensional data associated with traumatic brain injury (TBI) may enhance classification, as has been demonstrated with other fields such as cancer and image recognition. However, the correct internal consistency or normalization of these vector components will be required to allow appropriate comparison of the patient groupings or classification scheme. 


\section{SAATMAN ET AL.}

- There is a clear need for multivariable prognostic models which are validated and sensitive to therapeutic interventions. The IMPACT team has created the most recent, and perhaps the most comprehensive, of these models. They have largely limited themselves to models based on logistic regression which are meant to predict a global outcome measure, the GOS. These results provide an excellent basis for choosing classifiers (Maas et al., 2007; Murray et al., 2007). Older multivariable classifiers have included other logistic regression models (Narayan et al., 1981) and a classification tree model (Choi et al., 1988, 1991).

- It may be informative to apply regression models to examine the data for effect modifiers (interactions) and to determine the shape of the relationship of predictor to response. Other classification techniques which have not been tried, such as classification and regression tree, nearest neighbor clustering, or neural networks (Peto et al., 1976), may offer advantages over regression-based methods.

- The long-term solution will require application of techniques from statistics and bioinformatics. The resulting classification scheme can be both qualitative and quantitative. An example of a multiple vector-based analytical scheme is illustrated in Figure 3 . Vectors might include clinical exam, imaging studies, demographics, clinical course, genomics, and serum markers.

One additional area of interest raised at the workshop was that of misclassification at randomization due to delay in presentation of information. For example, a trial may be designed to exclude patients with an intraparenchymal hematoma. These hematomas can develop over time, so that a patient may have only a mild contusion at randomization but have a consolidated hematoma three days later. Although it might seem desirable to remove the patient from the study at this time, this is not acceptable for three reasons. First, in good trial design, only data that is collected prior to enrollment in the RCT should be used in the withdrawal decision-making process due to violations of entry criteria (Peto et al., 1976). Second, removing patients from a study following randomization is not acceptable to most regulatory agencies (Guidelines, 1988). Third and most importantly, there is no way to determine whether the treatment had any effect on the development of the hematoma. A better solution would be to develop classification models which predict the development of a delayed hematoma, and use this information at the time of randomization for patient clas- sification. Depending on the strength of the model, this approach will reduce the incidence of misclassification and can be taken for any condition which results in delayed presentation.

In conclusion, the development of tools to classify TBI will likely follow the classic "learn and confirm" paradigm found in most of science. The tools will initially be tried on an existing set of data, modifications will be made until the tool seems satisfactory, and then the tool will be applied to a new set of data and its utility assessed. To fully verify a new tool, advanced statistical methods, contemporary datasets, and well-constructed validation studies need to be available to and used by the TBI community.

\section{CONCLUSION}

As we have seen with other diseases such as cancer, improved classification systems have led to a better understanding of the mechanisms of disease and helped to refine treatments and improve outcome. The major conclusion from the workshop was that there is a need for a pathoanatomically based classification system for TBI if we are to successfully translate targeted therapies from the bench to the bedside. This does not diminish the importance of prognostic, etiologic, and symptom-based classification systems, which remain important for prevention, clinical management, and prediction of outcome in patients with TBI. The evaluation of targeted therapies for specific pathoanatomic lesions will likely require inclusion of less severely injured patients with more homogeneous injuries. Although this is a departure from traditional TBI clinical trial design, lessons learned from the study of these less complicated and more easily modeled injuries could then be applied to more severely injured patients.

A new TBI classification system for targeted therapies can be achieved within the next five years, but to do so will take a sustained and coordinated effort. Short-term efforts (12-18 months) that need to be undertaken include establishing several small, multidisciplinary working groups to review the literature and propose protocols for optimizing and standardizing TBI patient assessment. The working groups should be formed around the following topics: acute clinical assessment, neuroradiologic assessment, biomarkers, and functional outcomes assessment. An additional working group should be formed to identify resources and tools available for the development of a large, multidimensional database, including common data elements, data sharing, data mining, and bioinformatics. 
Review papers from these working groups would form the basis for the next step, which is to create a TBI data warehouse to facilitate multi-institutional collaboration and knowledge discovery. The database should be prospective, multidimensional, and inclusive of all severities of TBI and all ages. Given the very large numbers of people who sustain a TBI, it should be possible to enroll the necessary number of patients for the purpose of developing a new TBI classification system for targeted therapies within two years. These are not small tasks, and can only be achieved by a concerted, international effort. This workshop served primarily to start the process:

"Make no little plans; they have no magic to stir men's blood. . . . Make big plans . . . aim high in hope and work." (Daniel Burnham)

\section{APPENDIX: TBI CLASSIFICATION WORKSHOP AGENDA AND PARTICIPANT LIST}

Day 1-October 16, 2007

Background and Goals of the Workshop: Geoff Manley

Overview of Scientific Team Proposals for an Improved TBI Classification System

Moderator: David Hovda*32

Team Leaders: Alex Valadka, Andrew Maas, Ross Bullock

What is known about the heterogeneity of TBI and what are the major gaps in our knowledge?

Moderator: Geoff Ling*33

Panelists: Clay Goodman, Ross Bullock, Ewout Steyerberg

Which "tools" are recommended for discriminating between the heterogeneous TBI pathologies? What are the gaps and/or limitations?

Moderator: Douglas Smith*34

Panelists: David Wright, Juan Sahuquillo, Ramon Diaz-Arrastia

\section{Round Table Discussions by Topic and Expertise}

\section{Pathoanatomical Heterogeneity \& Animal Models} Moderator: David Hovda

Discussants: Tony Marmarou, Nikolous Plesnila, Pramod Dash, Linda Phillips, Frank Tortella*35, Leslie
Shupenko*16, Graham Teasdale, Ed Hall*1, John Povlishock*15

\section{Clinical Monitoring: Acute Phase}

Moderator: Walter Koroshetz*11

Discussants: David Wright, Daryl Gress*36, Neeraj

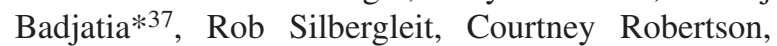
Nino Stocchetti, David Okonkwo, Gregory O'Shanick*38, Jam Ghajar*39

\section{Neuroimaging Tools}

Moderator: Debra Babcock*11

Discussants: Larry Latour*9 ${ }^{*}$, Dave Brody, Alisa Gean, Geoff Manley, Doug Smith, Tina Duhaime, Juan Sahuquillo

\section{Biomarkers}

Moderator: Joe Pancrazio*11

Discussants: Ron Hayes, Ramon Diaz-Arrastia, Kathy Saatman, Stephanie Fertig, Andrew Maas, Lawrence Marshall*40

\section{Clinical Monitoring: Outcomes}

Moderator: Ramona Hicks

Discussants: Lindsay Wilson, Wayne Gordon, Gordon Murray, Sandra Salan*41, Kathy Helmick*16, Louis French*42, Emmeline Edwards*11, Rebecca Desrocher*11, Ross Bullock, Alex Valadka, Nancy Temkin, Mark Ashley*43

\section{Data Management}

Moderator: Peter Gilbert*11

Discussants: Linda Papa, David Moore, Jean Langlois, Ewout Steyerberg, Hilaire Thompson, Charlie Contant, Clay Goodman

\section{Round Table Summary \& Recommendations}

Moderator: Doug Smith

Presentors: Walter Koroshetz, Debra Babcock, Joe Pancrazio, Wayne Gordon, Peter Gilbert, David Hovda

*Additional Participant Affiliations: ${ }^{32}$ University of California, Los Angeles, CA, ${ }^{33}$ Defense Advanced Research Projects Agency, Arlington, VA, ${ }^{34}$ University of Pennsylvania, Philadelphia, PA, ${ }^{35}$ Walter Reed Army Institute of Research, Silver Spring, MD, ${ }^{36}$ University of Virginia, Charlottesville, VA, ${ }^{37}$ Columbia University Medical Center, New York, NY, ${ }^{38}$ Brain Injury Association of America, McLean, VA, ${ }^{39}$ Brain Trauma Foundation, New York, NY, ${ }^{40}$ UCSD Medical Center, San Diego, CA, ${ }^{41}$ Social Security Administration, Office of Medical Policy, Washington, DC, ${ }^{42}$ Walter Reed Army Medical Center, Washington, DC, ${ }^{43}$ Centre for Neuro Skills, Bakersfield, CA. 


\section{SAATMAN ET AL.}

Day 2-October 17, 2007

What are the critical data elements and how should they be collected and analyzed to develop a TBI classification system for targeted interventions?

Moderator: David Hovda

Panelists: Lindsay Wilson, Linda Papa, Clay

Goodman

\section{Advisory Panel Summary and Recommendations \\ Moderator: Doug Smith \\ Panelists: Kathy Saatman and Members of Advisory Panel}

Action Plan - Geoff Manley and Ron Hayes

\section{ACKNOWLEDGMENTS}

We gratefully acknowledge the contributions that were made by all of the participants to the workshop discussions. Special thanks go to the session and round table moderators: Debra Babcock, Peter Gilbert, David Hovda, Walter Koroshetz, Geoffrey Ling, Joseph Pancrazio, and Douglas Smith. Support for the workshop came from the National Institute of Neurological Disorders and Stroke, the Brain Injury Association of America, the Defense and Veterans Brain Injury Consortium, and the National Institute on Disability and Rehabilitation Research. The views expressed here are those of the authors and do not represent those of the sponsoring institutions or the US Government.

\section{AUTHOR DISCLOSURE STATEMENT}

No conflicting financial interests exist.

\section{REFERENCES}

Adams, J.H., and Graham, D.I. (1994). An Introduction to Neuropathology. Churchill Livingstone: Edinburgh.

Adams, J.H., Graham, D.I., Murray, L.S., and Scott, G. (1982). Diffuse axonal injury due to nonmissile head injury in humans: an analysis of 45 cases. Ann. Neurol. 12, 557-563.

Adams, J.H., Mitchell, D.E., Graham, D.I., and Doyle, D. (1977). Diffuse brain damage of immediate impact type. Its relationship to "primary brain-stem damage" in head injury. Brain 100, 489-502.

Alderman, N., Burgess, P.W., Knight, C., and Henman, C. (2003). Ecological validity of a simplified version of the multiple errands shopping test. J. Int. Neuropsychol. Soc. 9, 31-44.
Armonda, R.A., Bell, R.S., Vo, A.H., Ling, G., DeGraba, T.J., Crandall, B., Ecklund, J., and Campbell, W.W. (2006). Wartime traumatic cerebral vasospasm: recent review of combat casualties. Neurosurgery 59, 1215-1225.

Baker, S.P., O’Neill, B., Haddon, W., Jr., and Long, W.B. (1974). The injury severity score: a method for describing patients with multiple injuries and evaluating emergency care. J. Trauma 14, 187-196.

Balestreri, M., Czosnyka, M., Chatfield, D.A., Steiner, L.A., Schmidt, E.A., Smielewski, P., Matta, B., and Pickard, J.D. (2004). Predictive value of Glasgow Coma Scale after brain trauma: change in trend over the past ten years. J. Neurol. Neurosurg. Psychiatry 75, 161-162.

Bouwens, S.F., Van Heugten, C.M., Aalten, P., Wolfs, C.A., Baarends, E.M., Van Menxel, D.A., and Verhey, F.R. (2008). Relationship between Measures of Dementia Severity and Observation of Daily Life Functioning as Measured with the Assessment of Motor and Process Skills (AMPS). Dement. Geriatr. Cogn. Disord. 25, 81-87.

Brihaye, J., Frowein, R., Lindgren, S., Loew, F., and Stroobandt, G. (1978). Report on the meeting of the W.F.N.S. neuro-traumatology committee, Brussels, 19-23 September 1976. Acta Neurochir. 40, 181-186.

Brown, M., Dijkers, M.P., Gordon, W.A., Ashman, T., Charatz, H., and Cheng, Z. (2004). Participation objective, participation subjective: a measure of participation combining outsider and insider perspectives. J. Head Trauma Rehabil. 19, 459-481.

Burgess, P.W., Alderman, N., Forbes, C., Costello, A., Coates, L.M., Dawson, D.R., Anderson, N.D., Gilbert, S.J., Dumontheil, I., and Channon, S. (2006). The case for the development and use of "ecologically valid" measures of executive function in experimental and clinical neuropsychology. J. Int. Neuropsychol. Soc. 12, 194-209.

Chambers, I.R., Barnes, J., Piper, I., Citerio, G., Enblad, P., Howells, T., Kiening, K., Matterns, J., Nilsson, P., Ragauskas, A., Sahuquillo, J., Yah, Y.H., and BrainIT Group. (2006). BrainIT: a trans-national head injury monitoring research network. Acta Neurochir. Suppl. 96, 7-10.

Champion, H.R., Sacco, W.J., Carnazzo, A.J., Copes, W., and Fouty, W.J. (1981). Trauma score. Crit. Care Med. 9, 672-676.

Choi, S.C., Muizelaar, J.P., Barnes, T.Y., Marmarou, A., Brooks, D.M. and Young, H.F. (1991). Prediction tree for severely head-injured patients. J. Neurosurg. 75, 251-255.

Choi, S.C., Narayan, R.K., Anderson, R.L. and Ward, J.D. (1988). Enhanced specificity of prognosis in severe head injury. J. Neurosurg. 69, 381-385.

Diener, E., Emmons, R.A., Larsen, R.J. and Griffin, S. (1985). The Satisfaction With Life Scale. J. Pers. Assess. 49, 71-75.

Doppenberg, E.M., Choi, S.C. and Bullock, R. (2004). Clinical trials in traumatic brain injury: lessons for the future. J. Neurosurg. Anesthesiol. 16, 87-94. 


\section{CLASSIFICATION OF TBI FOR TARGETED THERAPIES}

Fleischer, A.S., Payne, N.S. and Tindall, G.T. (1976). Continuous monitoring of intracranial pressure in severe closed head injury without mass lesions. Surg. Neurol. 6, 31-34.

Friedman, L.M., Furberg, C.D. and Demets, D.L. (1998). Fundamentals of Clinical Trials. Springer: New York.

Gabbe, B.J., Cameron, P.A. and Finch, C.F. (2003). The status of the Glasgow Coma Scale. Emerg. Med. (Fremantle. ) 15, 353-360.

Gallagher, C.N., Hutchinson, P.J. and Pickard, J.D. (2007). Neuroimaging in trauma. Curr. Opin. Neurol. 20, 403-409.

Gennarelli, T.A., Spielman, G.M., Langfitt, T.W., Gildenberg, P.L., Harrington, T., Jane, J.A., Marshall, L.F., Miller, J.D. and Pitts, L.H. (1982a). Influence of the type of intracranial lesion on outcome from severe head injury. J. Neurosurg. 56, 26-32.

Gennarelli, T.A. and Thibault, L.E. (1985). Biomechanics of Head Injury, in: Neurosurgery. R.H. Wilkins and S.S. Rengachary (eds), McGraw-Hill: New York, Pp. 1531-1536.

Gennarelli, T.A., Thibault, L.E., Adams, J.H., Graham, D.I., Thompson, C.J. and Marcincin, R.P. (1982). Diffuse axonal injury and traumatic coma in the primate. Ann. Neurol. 12, $564-574$.

Gerstenbrand, F. and Lucking, C.H. (1970). [Acute traumatic brainstem lesions]. Arch. Psychiatr. Nervenkr. 213, 264-281.

Graham, D.I., Adams, J.H., Murray, L.S., and Jennett, B. (2005). Neuropathology of the vegetative state after head injury. Neuropsychol. Rehabil. 15, 198-213.

Guideline for the format and content of the clinical and statistical sections of new drug applications. (1988). in: Center for Drug Evaluation and Research FDA, ed: Department of Health and Human Services.

Guidelines for the management of severe traumatic brain injury. (2007). J. Neurotrauma 24 Suppl 1:S1-106.

Jennett, B. and Bond, M. (1975). Assessment of outcome after severe brain damage. Lancet 1, 480-484.

Jennett, B., Snoek, J., Bond, M.R. and Brooks, N. (1981). Disability after severe head injury: observations on the use of the Glasgow Outcome Scale. J. Neurol. Neurosurg. Psychiatry 44, 285-293.

Kennedy, J.E., Jaffee, M.S., Leskin, G.A., Stokes, J.W., Leal, F.O. and Fitzpatrick, P.J. (2007). Posttraumatic stress disorder and posttraumatic stress disorder-like symptoms and mild traumatic brain injury. J. Rehabil. Res. Dev. 44, 895-920.

Kim, E., Lauterbach, E.C., Reeve, A., Arciniegas, D.B., Coburn, K.L., Mendez, M.F., Rummans, T.A., Coffey, E.C.; ANPA Committee on Research (2007). Neuropsychiatric complications of traumatic brain injury: a critical review of the literature (a report by the ANPA Committee on Research). J. Neuropsychiatry Clin. Neurosci. 19, 106-127.

Levin, H.S., High, W.M., Goethe, K.E., Sisson, R.A., Overall, J.E., Rhoades, H.M., Eisenberg, H.M., Kalisky, Z. and Gary,
H.E. (1987). The neurobehavioural rating scale: assessment of the behavioural sequelae of head injury by the clinician. J. Neurol. Neurosurg. Psychiatry 50, 183-193.

Lezak, M. D. and Malec, J. F. Mayo-Portland Adaptability Inventory. 2003. Rochester, MN, Mayo-Clinic (PMR-IDSMH).

Maas, A.I., Hukkelhoven, C.W., Marshall, L.F. and Steyerberg, E.W. (2005). Prediction of outcome in traumatic brain injury with computed tomographic characteristics: a comparison between the computed tomographic classification and combinations of computed tomographic predictors. Neurosurgery 57, 1173-1182.

Maas, A.I., Marmarou, A., Murray, G.D., Teasdale, S.G. and Steyerberg, E.W. (2007). Prognosis and clinical trial design in traumatic brain injury: the IMPACT study. J. Neurotrauma 24, 232-238.

Manley, G.T., Rosenthal, G., Lam, M., Morabito, D., Yan, D., Derugin, N., Bollen, A., Knudson, M.M. and Panter, S.S. (2006). Controlled cortical impact in swine: pathophysiology and biomechanics. J. Neurotrauma 23, 128-139.

Marmarou, A., Lu, J., Butcher, I., McHugh, G.S., Mushkudiani, N.A, Murray, G.D., Steyerberg, E.W. and Maas, A.I. (2007). IMPACT database of traumatic brain injury: design and description. J. Neurotrauma 24, 239-250.

Marshall, L.F. (2000). Head injury: recent past, present, and future. Neurosurgery 47, 546-561.

Marshall, L.F., Marshall, S.B., Klauber, M.R., Van Burkum Clark, M., Eisenberg, H., Jane, J.A., Luerssen, T.G., Marmarou, A. and Foulkes, M.A. (1992). The diagnosis of head injury requires a classification based on computed axial tomography. J. Neurotrauma 9 Suppl 1, S287-S292.

Medicine AftAoA. Joint Committee of the American Medical Association, the Society of Automotive Engineers and the American Association for Automotive Medicine. The Abbreviated Injury Scale (AIS). 1976. Morton Grove, IL, American Association for the Advancement of Automotive Medicine.

Medicine AftAoA. The Abbreviated Injury Scale, 1990 Revision. Association for the Advancement of Automotive Medicine. 15-24. 1990. Des Plaines, IL.

Merritt, B.K. and Fisher, A.G. (2003). Gender differences in the performance of activities of daily living. Arch. Phys. Med. Rehabil. 84, 1872-1877.

Murray, G.D., Butcher, I., McHugh, G.S., Lu, J., Mushkudiani, N.A., Maas, A.I., Marmarou, A. and Steyerberg, E.W. (2007). Multivariable prognostic analysis in traumatic brain injury: results from the IMPACT study. J. Neurotrauma 24, 329-337.

Narayan, R.K., Greenberg, R.P., Miller, J.D., Enas, G.G., Choi, S.C., Kishore, P.R., Selhorst, J.B., Lutz, H.A. $3^{\text {rd }}$ and Becker, D.P. (1981). Improved confidence of outcome prediction in severe head injury. A comparative analysis of the clinical ex- 


\section{SAATMAN ET AL.}

amination, multimodality evoked potentials, CT scanning, and intracranial pressure. J. Neurosurg. 54, 751-762.

Narayan, R.K., Michel, M.E., Ansell, B., Baethmann, A., Biegon, A., Bracken, M.B., Bullock, M.R., Choi, S.C., Clifton, G.L., Contant, C.F., Coplin, W.M., Dietrich, W.D., Ghajar, J., Grady, S.M., Grossman, R.G., Hall, E.D., Heetderks, W., Hovda, D.A., Jallo, J., Katz, R.L., Knoller, N., Kochanek, P.M., Maas, A.I., Majde, J., Marion, D.W., Marmarou, A., Marshall, L.F., McIntosh, T.K., Miller, E., Mohberg, N., Muizelaar, J.P., Pitts, L.H., Quinn, P., Riesenfeld, G., Robertson, C.S., Strauss, K.I., Teasdale, G., Temkin, N., Tuma, R., Wade, C., Walker, M.D., Weinrich, M., Whyte, J., Wilberger, J., Young, A.B. and Yurkewicz, L. (2002). Clinical trials in head injury. J. Neurotrauma 19, 503-557.

Oertel, M., Boscardin, W.J., Obrist, W.D., Glenn, T.C., McArthur, D.L., Gravori, T., Lee, J.H. and Martin, N.A. (2005). Posttraumatic vasospasm: the epidemiology, severity, and time course of an underestimated phenomenon: a prospective study performed in 299 patients. J. Neurosurg. 103, 812-824.

Peto, R., Pike, M.C., Armitage, P., Breslow, N.E., Cox, D.R., Howard, S.V., Mantel, N., McPherson, K., Peto, J. and Smith, P.G. (1976). Design and analysis of randomized clinical trials requiring prolonged observation of each patient. I. Introduction and design. Br. J. Cancer 34, 585-612.

Pineda, J.A., Lewis, S.B., Valadka, A.B., Papa, L., Hannay, H.J., Heaton, S.C., Demery, J.A., Liu, M.C., Aikman, J.M., Akle, V., Brophy, G.M., Tepas, J.J., Wang, K.K., Robertson, C.S. and Hayes, R.L. (2007). Clinical significance of alphaIIspectrin breakdown products in cerebrospinal fluid after severe traumatic brain injury. J. Neurotrauma 24, 354-366.

Piper, I., Citerio, G., Chambers, I., Contant, C., Enblad, P., Fiddes, H., Howells, T., Kiening, K., Nilsson, P., Yau, Y.H.; BrainIT Group. (2003). The BrainIT group: concept and core dataset definition. Acta Neurochir. (Wien.) 145, 615-628.

Povlishock, J.T. and Christman, C.W. (1995). The pathobiology of traumatically induced axonal injury in animals and humans: a review of current thoughts. J. Neurotrauma 12, $555-564$.

Povlishock, J.T., Hayes, R.L., Michel, M.E. and McIntosh, T.K. (1994). Workshop on animal models of traumatic brain injury. J. Neurotrauma 11, 723-732.

Povlishock, J.T. and Katz, D.I. (2005). Update of neuropathology and neurological recovery after traumatic brain injury. J. Head Trauma Rehabil. 20, 76-94.

Rappaport, M., Hall, K.M., Hopkins, K., Belleza, T. and Cope, D.N. (1982). Disability rating scale for severe head trauma: coma to community. Arch. Phys. Med. Rehabil. 63, 118-123.

Statler, K.D., Jenkins, L.W., Dixon, C.E., Clark, R.S., Marion, D.W. and Kochanek, P.M. (2001). The simple model versus the super model: translating experimental traumatic brain injury research to the bedside. J. Neurotrauma 18, 1195-1206.

Hukkelhoven C.W., Steyerberg E.W., Habbema J.D., Farace E., Marmarou A., Murray G.D., Marshall L.F., Maas A.I. (2005). Predicting outcome after traumatic brain injury: development and validation of a prognostic score based on admission characteristics. J Neurotrauma 22, 1025-1039.

Stocchetti, N., Pagan, F., Calappi, E., Canavesi, K., Beretta, L., Citerio, G., Cormio, M. and Colombo, A. (2004). Inaccurate early assessment of neurological severity in head injury. J. Neurotrauma 21, 1131-1140.

Strich, S.J. (1956). Diffuse degeneration of the cerebral white matter in severe dementia following head injury. J. Neurol. Neurosurg. Psychiatry 19, 163-185.

Strich, S.J. (1961). Shearing of nerve fibers as a cause of brain damage due to head injury; a pathological study of twenty cases. Lancet 2, 443-448.

Teasdale, G. and Jennett, B. (1974). Assessment of coma and impaired consciousness. A practical scale. Lancet 2, 81-84.

Thompson, H.J., Lifshitz, J., Marklund, N., Grady, M.S., Graham, D.I., Hovda, D.A. and McIntosh, T.K. (2005). Lateral fluid percussion brain injury: a 15-year review and evaluation. J. Neurotrauma 22, 42-75.

Van Beek, J.G., Mushkudiani, N.A., Steyerberg, E.W., Butcher, I., McHugh, G.S., Lu, J., Marmarou, A., Murray, G.D. and Maas, A.I. (2007). Prognostic value of admission laboratory parameters in traumatic brain injury: results from the IMPACT study. J. Neurotrauma 24, 315-328.

Waehrens, E.E. and Fisher, A.G. (2007). Improving quality of ADL performance after rehabilitation among people with acquired brain injury. Scand. J. Occup. Ther. 22, 1-8.

Warden, D. (2006). Military TBI during the Iraq and Afghanistan wars. J. Head Trauma Rehabil. 21, 398-402.

Wijdicks, E.F., Bamlet, W.R., Maramattom, B.V., Manno, E.M. and McClelland, R.L. (2005). Validation of a new coma scale: The FOUR score. Ann. Neurol. 58, 585-593.

World Health Organization. International Classification of Functioning, Disability and Health. 2001. Geneva: WHO.

Address reprint requests to: Geoffrey T. Manley, M.D., Ph.D. Department of Neurological Surgery University of California, San Francisco 1001 Potrero Avenue, Room 101 San Francisco, CA 94110

E-mail: manleyg@neurosurg.ucsf.edu 